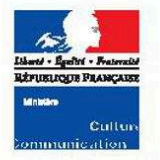

Secrétariat général

\title{
Délégation
}

au développement

et aux affaires

internationales

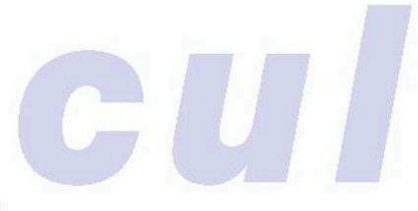

des études,

de la prospective
et des statistiques

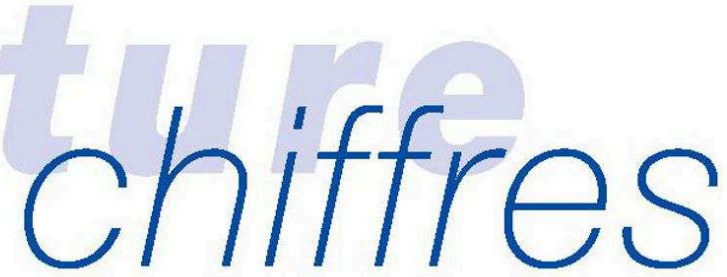

82, rue Saint-Honoré, 75033 Paris cedex 0

줄 0140157913 - 国 0140157999

\section{Statistiques d'entreprises des industries culturelles}

\section{Valérie Deroin*}

Le secteur des industries culturelles qui relève des activités de services constitue un sous-ensemble du secteur des industries de l'information et de la communication ${ }^{1}$. Il regroupe des fonctions de conception, de création et de fabrication, de distribution ainsi que de commercialisation de supports physiques reproductibles ou de communication.

En 2006 , il a réalisé $7,3 \%$ du chiffre d'affaires des services marchands, soit 43 milliards d'euros, et a employé $3,8 \%$ des effectifs salariés, soit 157000 salariés (effectif salarié moyen annuel, équivalent temps plein $^{2}$ )

Secteur d'oligopoles à frange concurrentielle, sa structure est conditionnée par les modèles d'affaires de ses entreprises : des coûts fixes de production relativement élevés et des coûts variables de reproduction ou diffusion relativement faibles favorisent la concentration industrielle dans le cadre de marchés caractérisés par une demande variable et imprévisible de produits qualifiés de biens d'expérience.

\section{REPÈRES ÉCONOMIQUES SUR LE SECTEUR DES INDUSTRIES CULTURELLES}

\section{Population des entreprises:} 23450 entreprises en 2006

D'un point de vue économique et statistique, le industries culturelles concernent trois catégories principales d'activités économiques :

- les activités d'édition qui comprennent l'édition de livres, de journaux, de revues et périodiques ainsi que l'édition d'enregistrements sonores;

- les activités audiovisuelles qui comprennent la production de films (pour la télévision et le cinéma) et de programmes de télévision, les activités de distribution (cinéma, vidéo, bouquets de programmes, radio), les activités d'édition de chaînes généralistes comme thématiques, de vidéo, et enfin les activités de projection ou de prestations techniques pour le cinéma et la télévision :

- les activités des agences de presse.

Économiquement, au regard des modes de financement des industries culturelles et de communication, l'activité du secteur de la publicité doit aussi être observée, qu'il s'agisse de la gestion des supports

\footnotetext{
*Attachée statisticienne de l'Insee.
1. Voir l'encadré « Approche slatistique sectorielle des industries culturelles », p. 5 .

2. Effectif salarie moyen en equivalent temps plein (ETP) qui tient compte du caractère saisonnier de l'emploi et permet des comparaisons entre secteurs. L'équivalent temps plein représente le nombre total d'heures travaillées dans l'activité considerée, divisé par la moyenne annuelle

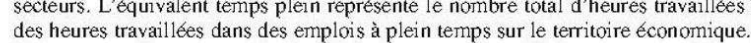

des heures travaillées dans des emplois à plein temps sur le territoire économique. culture, à paraître dans la collection «Culture chiffres» 2009-1, janvier 2009) qui a recours à un concept plus large d'actifs déclarés sur une base minimale de 1 heure d'emploi.
} 


\section{Statistiques d'entreprises des industries culturelles}

\section{Valérie Deroin}

Éditeur : Département des études, de la prospective et des statistiques

Lieu d'édition : paris

Année d'édition : 2008

Date de mise en ligne : 21 septembre 2015

Collection : Culture chiffres

ISBN électronique : 9782111398405

\section{Sbooks}

http://books.openedition.org

\section{Édition imprimée}

Date de publication : 1 décembre 2008

Nombre de pages : 8

\section{Référence électronique}

DEROIN, Valérie. Statistiques d'entreprises des industries culturelles. Nouvelle édition [en ligne]. paris: Département des études, de la prospective et des statistiques, 2008 (généré le 25 avril 2021).

Disponible sur Internet : <http://books.openedition.org/deps/777>. ISBN : 9782111398405 

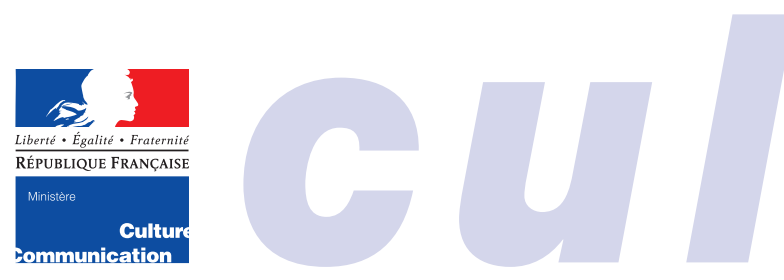

Secrétariat général

Délégation

au développement

et aux affaires

internationales

Département

des études,

de la prospective

et des statistiques

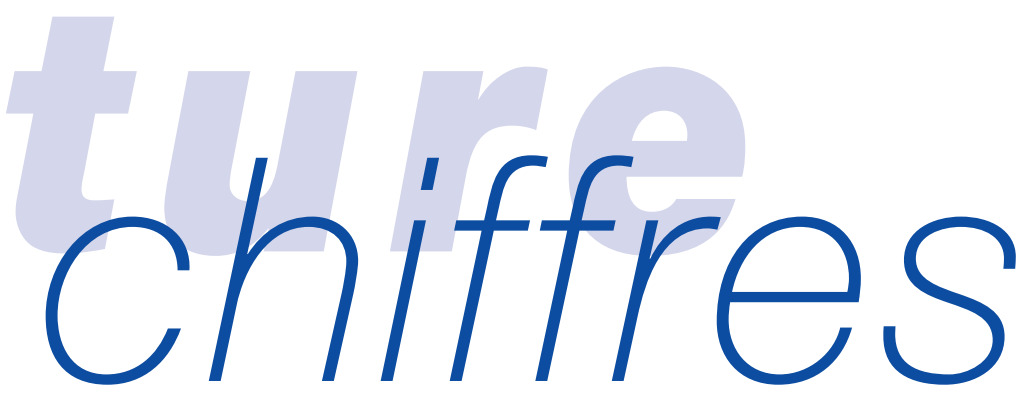

\section{Statistiques d'entreprises des industries culturelles}

\section{Valérie Deroin*}

Le secteur des industries culturelles qui relève des activités de services constitue un sous-ensemble du secteur des industries de l'information et de la communication ${ }^{1}$. Il regroupe des fonctions de conception, de création et de fabrication, de distribution ainsi que de commercialisation de supports physiques reproductibles ou de communication.

En 2006, il a réalisé 7,3\% du chiffre d'affaires des services marchands, soit 43 milliards d'euros, et a employé 3,8\% des effectifs salariés, soit 157000 salariés (effectif salarié moyen annuel, équivalent temps plein $^{2}$ ).

Secteur d'oligopoles à frange concurrentielle, sa structure est conditionnée par les modèles d'affaires de ses entreprises : des coûts fixes de production relativement élevés et des coûts variables de reproduction ou diffusion relativement faibles favorisent la concentration industrielle dans le cadre de marchés caractérisés par une demande variable et imprévisible de produits qualifiés de biens d'expérience.
REPÈRES ÉCONOMIQUES

\section{SUR LE SECTEUR DES INDUSTRIES \\ CULTURELLES}

\section{Population des entreprises : 23450 entreprises en 2006}

D'un point de vue économique et statistique, les industries culturelles concernent trois catégories principales d'activités économiques :

- les activités d'édition qui comprennent l'édition de livres, de journaux, de revues et périodiques ainsi que l'édition d'enregistrements sonores ;

- les activités audiovisuelles qui comprennent la production de films (pour la télévision et le cinéma) et de programmes de télévision, les activités de distribution (cinéma, vidéo, bouquets de programmes, radio), les activités d'édition de chaînes généralistes comme thématiques, de vidéo, et enfin les activités de projection ou de prestations techniques pour le cinéma et la télévision;

- les activités des agences de presse.

Économiquement, au regard des modes de financement des industries culturelles et de communication, l'activité du secteur de la publicité doit aussi être observée, qu'il s'agisse de la gestion des supports

\footnotetext{
* Attachée statisticienne de l'Insee.

1. Voir l'encadré « Approche statistique sectorielle des industries culturelles», p. 5.

2. Effectif salarié moyen en équivalent temps plein (ETP) qui tient compte du caractère saisonnier de l'emploi et permet des comparaisons entre secteurs. L'équivalent temps plein représente le nombre total d'heures travaillées dans l'activité considérée, divisé par la moyenne annuelle des heures travaillées dans des emplois à plein temps sur le territoire économique.

Remarque : Cette mesure de l'emploi n'est pas celle utilisée pour comptabiliser l'emploi salarié dans la culture (L'emploi salarié dans la culture, à paraître dans la collection «Culture chiffres » 2009-1, janvier 2009) qui a recours à un concept plus large d'actifs déclarés sur une base minimale de 1 heure d'emploi.
} 
Graphique 1 - Population des entreprises en 2006 par catégories d'activités économiques

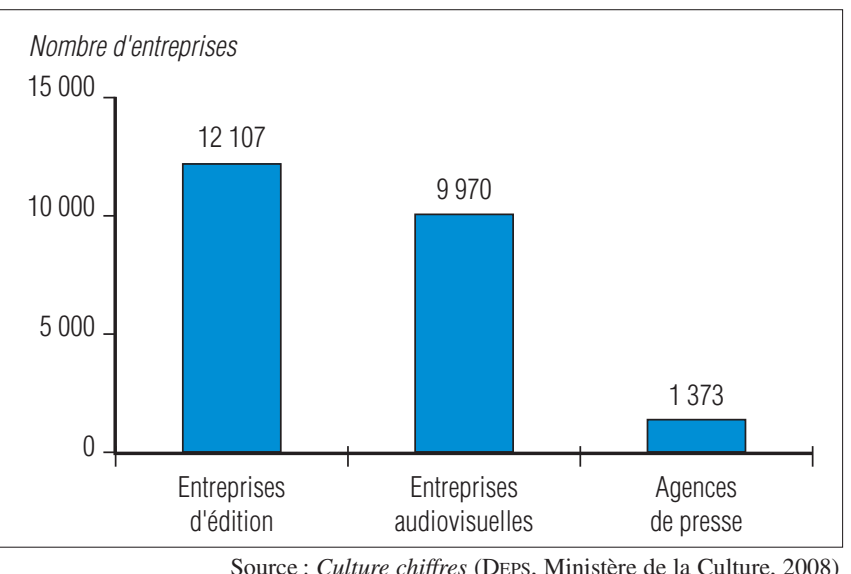

de publicité ou des agences et entreprises de conseil en publicité. La publicité finance en effet une partie importante des activités de ces industries. Ainsi, en 2008, $30 \%$ des 32,7 milliards d'euros d'investissements des annonceurs ont été consacrés aux médias des industries culturelles, dont $13 \%$ à la presse et $13 \%$ à la télévision ${ }^{3}$.

\section{Emploi : 157000 salariés (équivalent ETP) en 2006}

\section{Le non-salariat caractérise l'emploi dans le secteur}

$53 \%$ des entreprises des industries culturelles n'ont pas de salarié, le chef d'entreprise étant dans ce cas la seule personne déclarée travaillant dans l'en-
Graphique 2-Répartition du nombre de salariés par catégories d'activités économiques

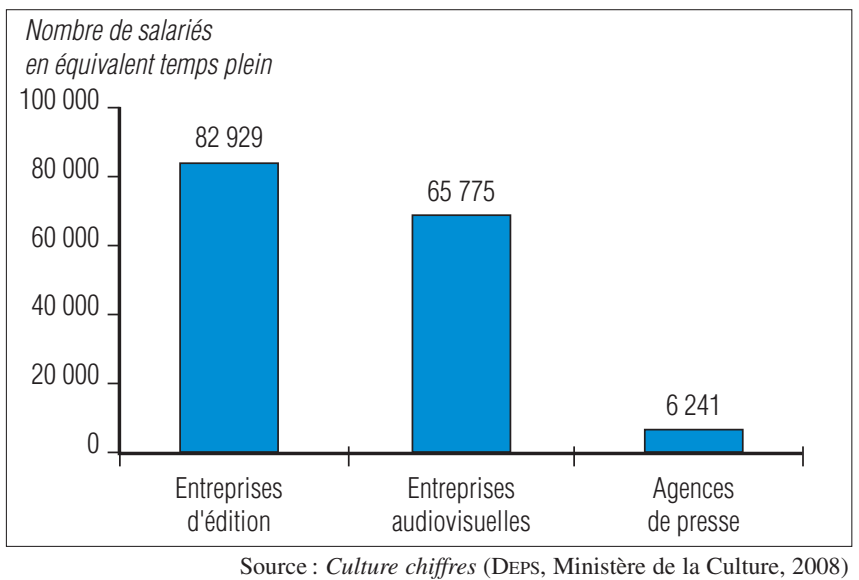

treprise (tableau 1). Dans l'ensemble du secteur des services, le nombre d'entreprises sans salarié n'est que de $34 \%$.

Cette particularité des industries culturelles, liées à des emplois temporaires et de courte durée, varie selon les secteurs. L'audiovisuel se rapproche de l'ensemble des services, avec seulement $38 \%$ d'entreprises sans salarié, et la situation varie d'une activité audiovisuelle à l'autre. Le non-salariat est important dans le cinéma et la vidéo ( $41 \%$ d'effectif sans salarié), il concerne un peu moins d'un tiers des entreprises télévisuelles (29\%), tandis que la radio est dominée par l'emploi salarié (seulement $9 \%$ sans salarié). L'édition, avec un taux élevé de $64 \%$ d'entreprises sans salarié, et les agences de presse $(58 \%)$ reposent sur l'entreprise individuelle basée sur l'emploi non salarié.

Tableau 1 - Répartition du nombre d'entreprises de moins de 20 salariés en 2006

\begin{tabular}{|c|c|c|c|c|c|c|c|c|}
\hline & \multicolumn{8}{|c|}{ Répartition des entreprises selon le nombre de salariés } \\
\hline & Total & $\begin{array}{c}\text { Hors } \\
\text { tranche }\end{array}$ & 0 & 1 ou 2 & 3 à 5 & 6 à 9 & 10 à 19 & $\begin{array}{c}20 \\
\text { ou plus }\end{array}$ \\
\hline Industries culturelles $(A+B+C)$ & $(23450)$ & 6 & 53 & 18 & 10 & 5 & 4 & 4 \\
\hline $\begin{array}{l}\text { Édition }(\mathrm{A}) \\
\text { Édition de livres } \\
\text { Édition de journaux } \\
\text { Édition de revues et périodiques } \\
\text { Édition d'enregistrements sonores }\end{array}$ & $\begin{array}{r}(12107) \\
(3324) \\
(1594) \\
(4159) \\
(3030)\end{array}$ & $\begin{array}{l}\text { n.d. } \\
\text { n.d. } \\
\text { n.d. } \\
\text { n.d. } \\
\text { n.d. }\end{array}$ & $\begin{array}{l}64 \\
64 \\
58 \\
56 \\
78\end{array}$ & $\begin{array}{l}16 \\
16 \\
17 \\
16 \\
15\end{array}$ & $\begin{array}{r}8 \\
8 \\
9 \\
10 \\
4\end{array}$ & $\begin{array}{l}5 \\
5 \\
5 \\
7 \\
2\end{array}$ & $\begin{array}{l}3 \\
3 \\
4 \\
5 \\
1\end{array}$ & $\begin{array}{r}4 \\
4 \\
7 \\
6 \\
\text { n.s. }\end{array}$ \\
\hline $\begin{array}{l}\text { Activités audiovisuelles (B) } \\
\text { Cinéma et vidéo } \\
\text { Radio } \\
\text { Télévision }\end{array}$ & $\begin{array}{r}9970 \\
8648 \\
613 \\
709\end{array}$ & $\begin{array}{l}14 \\
13 \\
35 \\
16\end{array}$ & $\begin{array}{r}38 \\
41 \\
9 \\
29\end{array}$ & $\begin{array}{l}20 \\
20 \\
27 \\
14\end{array}$ & $\begin{array}{l}11 \\
11 \\
13 \\
13\end{array}$ & $\begin{array}{l}6 \\
5 \\
9 \\
8\end{array}$ & $\begin{array}{l}5 \\
5 \\
3 \\
7\end{array}$ & $\begin{array}{r}5 \\
4 \\
5 \\
15\end{array}$ \\
\hline Agences de presse $(C)$ & 1373 & 6 & 59 & 12 & 16 & 3 & 2 & 3 \\
\hline Publicité & 18371 & 4 & 32 & 31 & 18 & 8 & 4 & 4 \\
\hline Ensemble des services & 838427 & 5 & 34 & 30 & 17 & 7 & 4 & 3 \\
\hline
\end{tabular}




\section{Chiffre d'affaires : \\ 43 milliards d'euros en 2006}

\section{Une croissance modérée des industries culturelles depuis 2003}

De 2003 à 2006, la croissance du chiffre d'affaires et du nombre de salariés (effectif salarié moyen ETP) des entreprises culturelles a été inférieure à l'ensemble des services : alors que la croissance du chiffre d'affaires des services a progressé de $20 \%$ sur la période, celle des industries culturelles a été de $5 \%$; l'effectif salarié des services a gagné $8 \%$ tandis que celui des industries culturelles restait constant (graphique 4).

En revanche, le nombre d'entreprises a augmenté de $17 \%$ sur la période, soit un peu plus que pour l'ensemble des services : ceci est essentiellement le fait de créations de très petites voire de micro-entreprises alors que, parallèlement, le phénomène de concentration des grandes entreprises s'est poursuivi et que le nombre d'entreprises de 20 salariés et plus des industries culturelles a diminué.

\section{Graphique 3 - Répartition du chiffre d'affaires par catégories d'activités économiques}

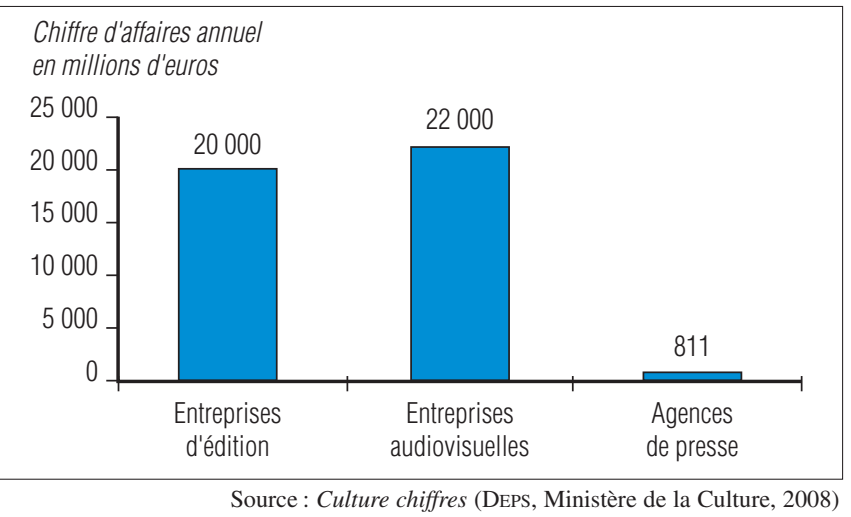

\section{Graphique 4-Évolution du chiffre d'affaires, du nombre d'entreprises et de l'effectif salarié moyen ETP de 2003 à 2006}

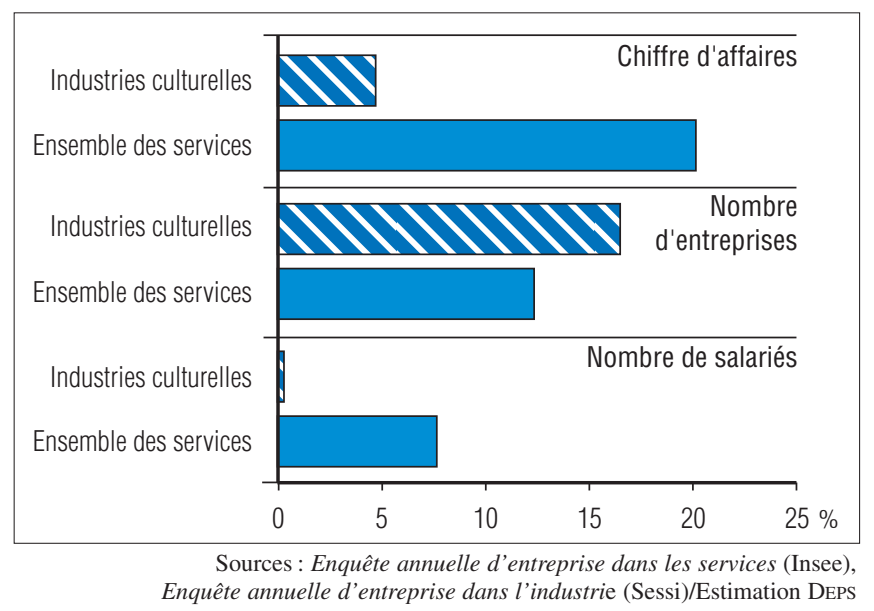

\section{UN SECTEUR TRÈS CONCENTRÉ}

Le secteur se caractérise par la taille modeste de la plupart des entreprises et par une forte concentration, mesurée par la répartition du chiffre d'affaires total entre les entreprises de 20 salariés et plus et les autres.

Les 1000 entreprises de 20 salariés ou plus des industries culturelles représentent, en nombre, $4 \%$ des entreprises et concentrent $81 \%$ du chiffre d'affaires alors que, par comparaison, celles des services marchands $(3 \%)$ ne produisent que $59 \%$ du chiffre d'affaires (graphiques 5 et 6 ).

La concentration est plus importante dans le secteur de l'édition où $4 \%$ des entreprises de plus de 20 salariés représentent $71 \%$ de l'effectif salarié moyen et réalisent $84 \%$ du chiffre d'affaires (tableau 3) que pour les activités audiovisuelles. Les entreprises audiovisuelles se différencient selon les secteurs : les activités de la télévision comptent $15 \%$ d'entreprises de plus de 20 salariés qui génèrent $93 \%$ du chiffre d'affaires, tandis que dans le cinéma et la vidéo, $4 \%$ d'entreprises de 20 salariés ou plus produisent $59 \%$ du chiffre d'affaires du secteur.

\section{Graphique 5 - Structure comparée des industries culturelles et des services en 2006 : répartition du nombre d'entreprises selon le nombre de salariés}

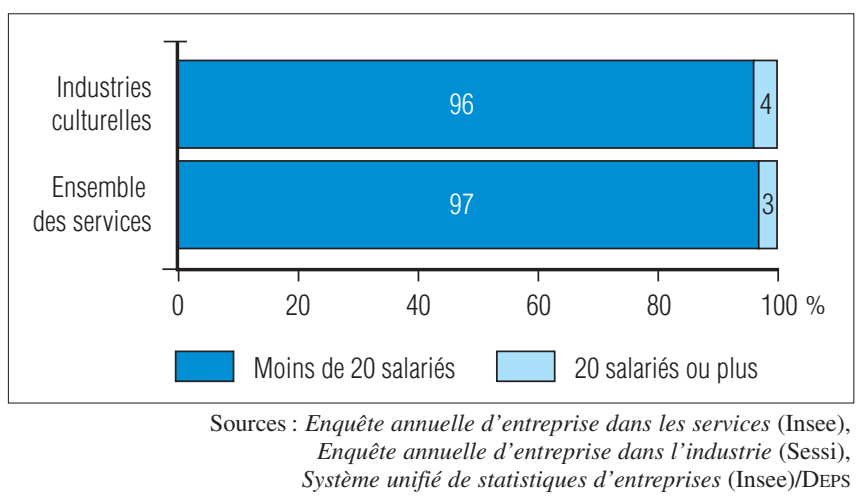

\section{Graphique 6 - Structure comparée des industries culturelles et des services en 2006: répartition du chiffre d'affaires selon le nombre de salariés}

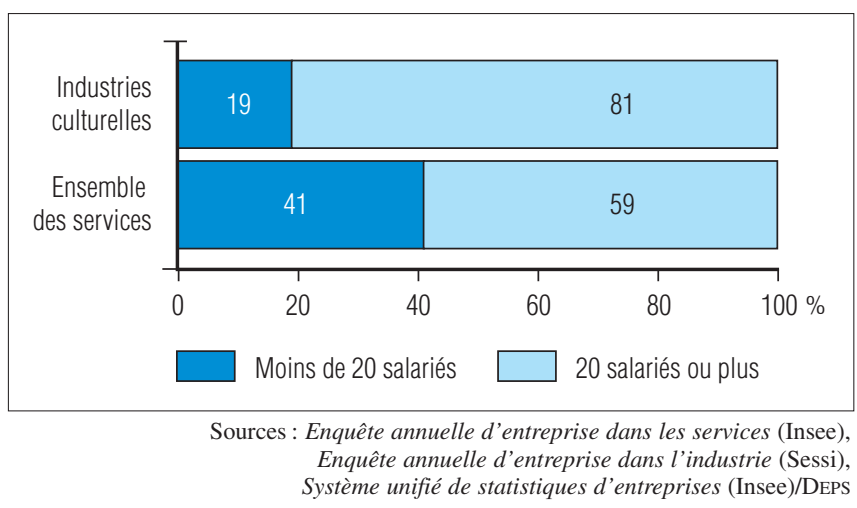




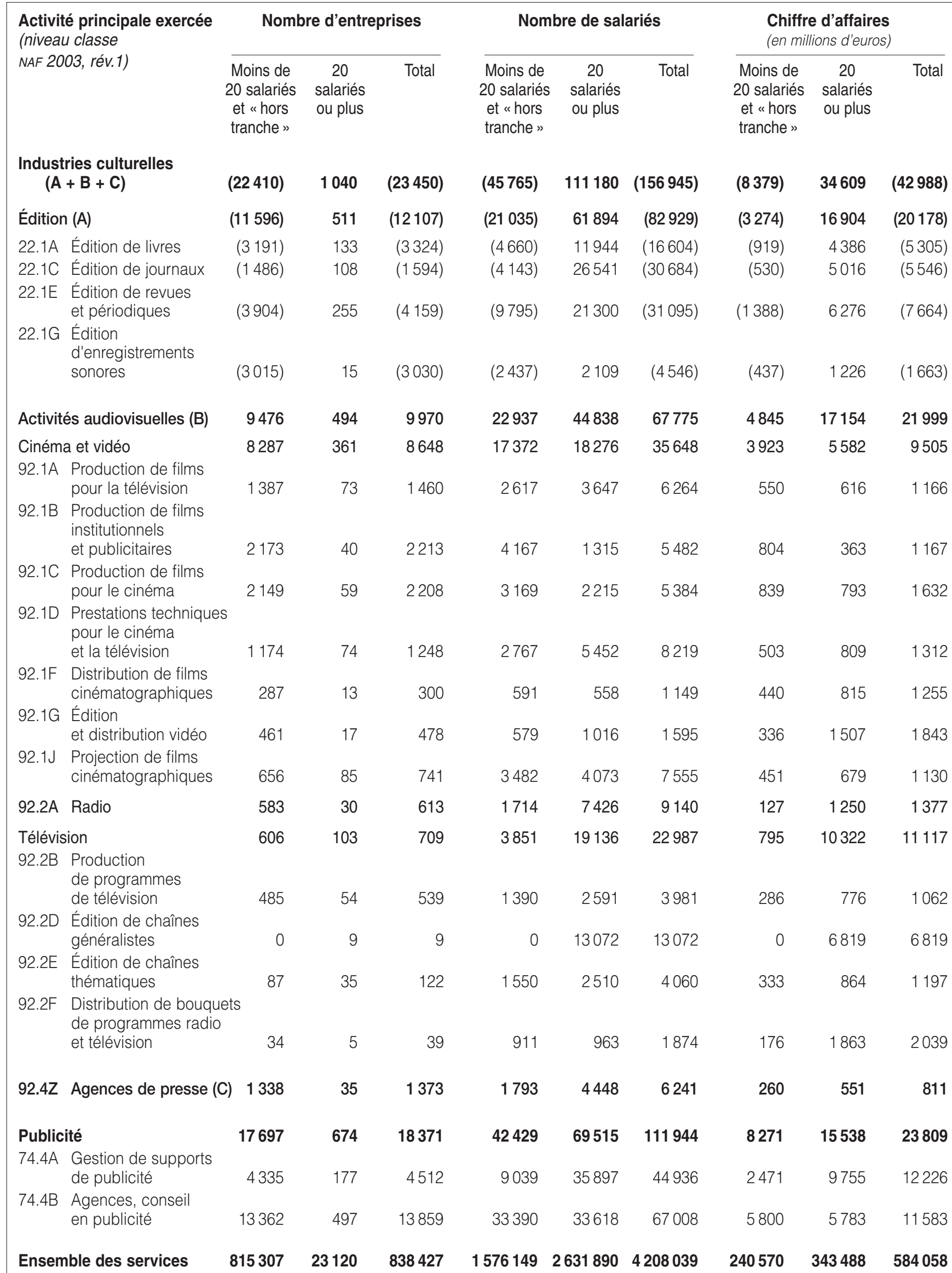

$N B$ : les chiffres entre parenthèses sont des estimations. Le champ de l'enquête annuelle dans l'industrie ne couvrant pas les entreprises de moins de 20 salariés, les résultats de l'édition ont été estimés à partir du répertoire Sirene ainsi que des tableaux diffusés dans I'Insee résultats $n^{\circ} 36$, Économie, «Images économiques des entreprises et des groupes 2006 ".

Sources : Enquête annuelle d'entreprise dans les services (Insee), Enquête annuelle d'entreprise dans l'industrie (Sessi), Système unifié de statistiques d'entreprises (Insee)/Deps 


\section{Approche statistique sectorielle du champ des industries culturelles}

Le secteur des industries culturelles et de communication regroupe les activités d'édition (livres, disques, presse nomenclatures d'activités 22.1A, 22.1C, 22.1E, 22.1G), les activités audiovisuelles (cinéma et vidéo, radio, télévision - nomenclatures d'activités $92.1 \mathrm{~A}$ à $92.1 \mathrm{~J}, 92.2 \mathrm{~A}$, et $92.2 \mathrm{~B}$ à $92.2 \mathrm{~F}$ ) et les agences de presse (nomenclature d'activités 92.4Z).

L'analyse statistique développée ici intègre les agences de presse, qui, comme les activités audiovisuelles, font partie des services aux particuliers : elles collectent, synthétisent et diffusent les informations écrites, photographiques et audiovisuelles utilisées par la presse et les autres médias. Ainsi, elles apportent une valeur ajoutée à l'information en termes de contenus et regroupent les activités et emplois des journalistes et des photographes. Les activités des agences de publicité et de gestion des supports de publicité, activités de services aux entreprises, sont économiquement liées aux industries d'information et de communication. L'approche retenue pour l'analyse statistique du champ des industries culturelles s'appuie sur la nomenclature d'activités française (NAF 2003, rév.1) qui détermine le classement des entreprises selon leur activité principale. Cependant, si la nomenclature 2003 comprend le multimédia, elle ne permet pas encore de l'isoler*. La nouvelle nomenclature d'activités française révision 2 (NAF

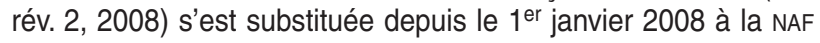
rév. 1 de 2003. Le champ des industries culturelles y est intégré à la nouvelle section $\mathbf{J}$ " Information et communication " qui regroupe les activités de production et de distribution de l'information et des produits culturels, la mise à disposition de moyens permettant de transmettre ou de distribuer ces produits ainsi que les données ou les communications. Cette section contient les activités d'édition, qui sont donc rapprochées des autres activités de services comme la production de films cinématographiques, de vidéos et de programmes de télévision, ainsi que la programmation et la diffusion de chaînes de radio et de télévision, les télécommunications, les activités informatiques ou encore les autres services d'information dont les agences de presse et les portails internet.

* Le champ de l'analyse est contraint par la disponibilité d'informations homogènes : ainsi, le commerce de disque, de livre et de presse n'a pas pu être intégré au champ, de même que les entreprises de l'imprimerie et de reproduction d'enregistrements sonores, considérées comme des activités connexes, ou les activités de banques de données.

\section{Radio et télévision : une concentration plus marquée encore}

En 2006, les entreprises de 20 salariés ou plus du cinéma et de la vidéo réalisent $59 \%$ du chiffre d'affaires du secteur (tableau 3), soit un taux similaire à celui des entreprises de l'ensemble des services. Toutefois, le sous-secteur du cinéma et de la vidéo est très contrasté : le chiffre d'affaires de la production de films institutionnels et publicitaires n'est réalisé qu'à hauteur de $31 \%$ par des entreprises de 20 salariés ou plus; sa concentration est donc plus faible que celle de l'édition et de la distribution vidéo ( $82 \%$ du chiffre d'affaires des entreprises de 20 salariés ou plus).

La concentration est forte dans la radio $(91 \%$ du chiffre d'affaires réalisé par les 20 salariés ou plus) et dans les entreprises de la télévision (93\%). De fait, les activités télévisuelles sont concentrées entre les mains d'une dizaine d'entreprises (chaînes et bouquets) qui réalisent l'essentiel du chiffre d'affaires global.

La structure sectorielle des agences de presse est la moins polarisée : les entreprises de plus de 20 salariés ne représentent en effet que $3 \%$ du nombre d'entreprises et réalisent $68 \%$ du chiffre d'affaires.

\section{DES TAUX DE VALEUR AJOUTÉE VARIABLES SELON LES ACTIVITÉS DES INDUSTRIES CULTURELLES ET DE COMMUNICATION ${ }^{4}$}

Le taux de valeur ajoutée ${ }^{5}$ est le rapport de la valeur ajoutée sur le chiffre d'affaires. Il permet de mesurer la capacité de l'entreprise à créer de la valeur ajoutée par rapport au chiffre d'affaires réalisé. Mesure du poids des consommations intermédiaires

Tableau 3 - Répartition des entreprises de 20 salariés ou plus en 2006

\begin{tabular}{|c|c|c|c|c|c|c|}
\hline \multirow{2}{*}{$\begin{array}{l}\text { Activité principale exercée } \\
\text { (niveau classe NAF 2003, rév.1) }\end{array}$} & \multicolumn{3}{|c|}{ Ensemble des entreprises } & \multicolumn{3}{|c|}{ Entreprises de $\mathbf{2 0}$ salariés ou plus (en \%) } \\
\hline & $\begin{array}{c}\text { Nombre } \\
\text { d'entreprises }\end{array}$ & $\begin{array}{c}\text { Nombre } \\
\text { de salariés }\end{array}$ & $\begin{array}{l}\text { Chiffre d'affaires } \\
\text { (millions d'euros) }\end{array}$ & $\begin{array}{c}\text { Nombre } \\
\text { d'entreprises }\end{array}$ & $\begin{array}{c}\text { Nombre } \\
\text { de salariés }\end{array}$ & $\begin{array}{l}\text { Chiffre d'affaires } \\
\text { (millions d'euros) }\end{array}$ \\
\hline Industries culturelles $(A+B+C)$ & $(23450)$ & (156 945) & (42 988) & 4 & 71 & 81 \\
\hline Édition (livres, disques, presse) (A) & (12 107) & (82 929) & $(20178)$ & 4 & 75 & 84 \\
\hline $\begin{array}{l}\text { Activités audiovisuelles (B) } \\
\text { Cinéma et vidéo } \\
\text { Radio } \\
\text { Télévision }\end{array}$ & $\begin{array}{r}9970 \\
8648 \\
613 \\
709\end{array}$ & $\begin{array}{r}67775 \\
35648 \\
9140 \\
22987\end{array}$ & $\begin{array}{r}21999 \\
9505 \\
1377 \\
11117\end{array}$ & $\begin{array}{r}5 \\
4 \\
5 \\
15\end{array}$ & $\begin{array}{l}66 \\
51 \\
81 \\
83\end{array}$ & $\begin{array}{l}78 \\
59 \\
91 \\
93\end{array}$ \\
\hline Agences de presse $(\mathrm{C})$ & 1373 & 6241 & 811 & 3 & 71 & 68 \\
\hline Publicité & 18371 & 111944 & 23809 & 4 & 62 & 65 \\
\hline Ensemble des services & 838427 & 4208039 & 584058 & 3 & 63 & 59 \\
\hline
\end{tabular}

Sources: Enquête annuelle d'entreprise dans les services (Insee), Enquête annuelle d'entreprise dans l'industrie (Sessi), REE (Répertoire des entreprises et des établissements) - Sirene (Système informatique pour le répertoire des entreprises et de leurs établissements) (Insee)/DEPS

4. Données 2006.

5. Valeur ajoutée (VA) : différence entre la valeur de la production d'une entreprise (chiffre d'affaires ou recette) et la valeur de tous les facteurs de production achetés aux autres entreprises (achats de biens intermédiaires ou coût des consommations intermédiaires). 
dans le processus de production, ce solde analyse la valeur concrètement créée par l'entreprise sur l'année. Il permet notamment de payer les frais de personnel (salaires et cotisations sociales), le fisc (impôts liés à la production, sur les sociétés et sur le patrimoine) ainsi que les actionnaires (dividendes).

Globalement, les industries culturelles ont un taux de valeur ajoutée $(41 \%)$ assez proche mais inférieur à la moyenne de l'ensemble des services marchands (50\%), et plus particulièrement des services aux particuliers (45\%).

Parmi les industries culturelles, ce taux est cependant variable selon les catégories d'activités. Il est plus faible dans l'édition (livres, disques, presse) : $36 \%$, que dans les activités audiovisuelles : $47 \%$. Ce taux moyen masque encore des différences selon les types d'activités audiovisuelles : $58 \%$ pour la radio, $53 \%$ pour le cinéma et la vidéo et $41 \%$ pour la télévision. Enfin, les activités des agences de presse se caractérisent par une forte valeur ajoutée : $61 \%$ (graphique 7).

\section{Graphique 7 - Part de la valeur ajoutée en 2006 par activités}

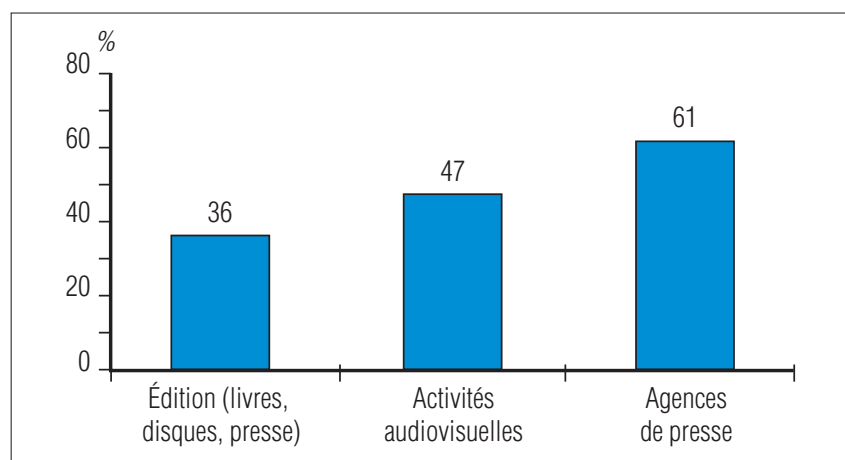

$N B$ : les chiffres entre parenthèses sont des estimations.

Sources : Enquête annuelle d'entreprise dans les services (Insee), Enquête annuelle d'entreprise dans l'industrie (Sessi)/DEPS

\section{L'audiovisuel : un ensemble dynamique à forte valeur ajoutée}

Les activités audiovisuelles (cinéma, vidéo, radio et télévision), dont le nombre d'entreprises a doublé depuis 1993, ont stabilisé leur développement entre 2003 et 2006. Comparées à l'ensemble des services marchands, les activités audiovisuelles se distinguent par une forte valeur ajoutée par personne occupée : la moyenne de l'audiovisuel, avec 152000 euros, est presque trois fois supérieure à celle des services (59 000 euros) (graphique 8). Ces résultats sont en particulier le fait des secteurs de la distribution (cinéma, vidéo et télévision): l'édition de chaînes généralistes génère une valeur ajoutée par personne occupée de 249000 euros et l'édition et la distribution vidéo de 273000 euros. Pour comparaison, celle de la projection de films cinématographiques est de 53000 euros seulement.

\section{Graphique 8 - Valeur ajoutée au prix du marché par personne occupée dans l'audiovisuel"}

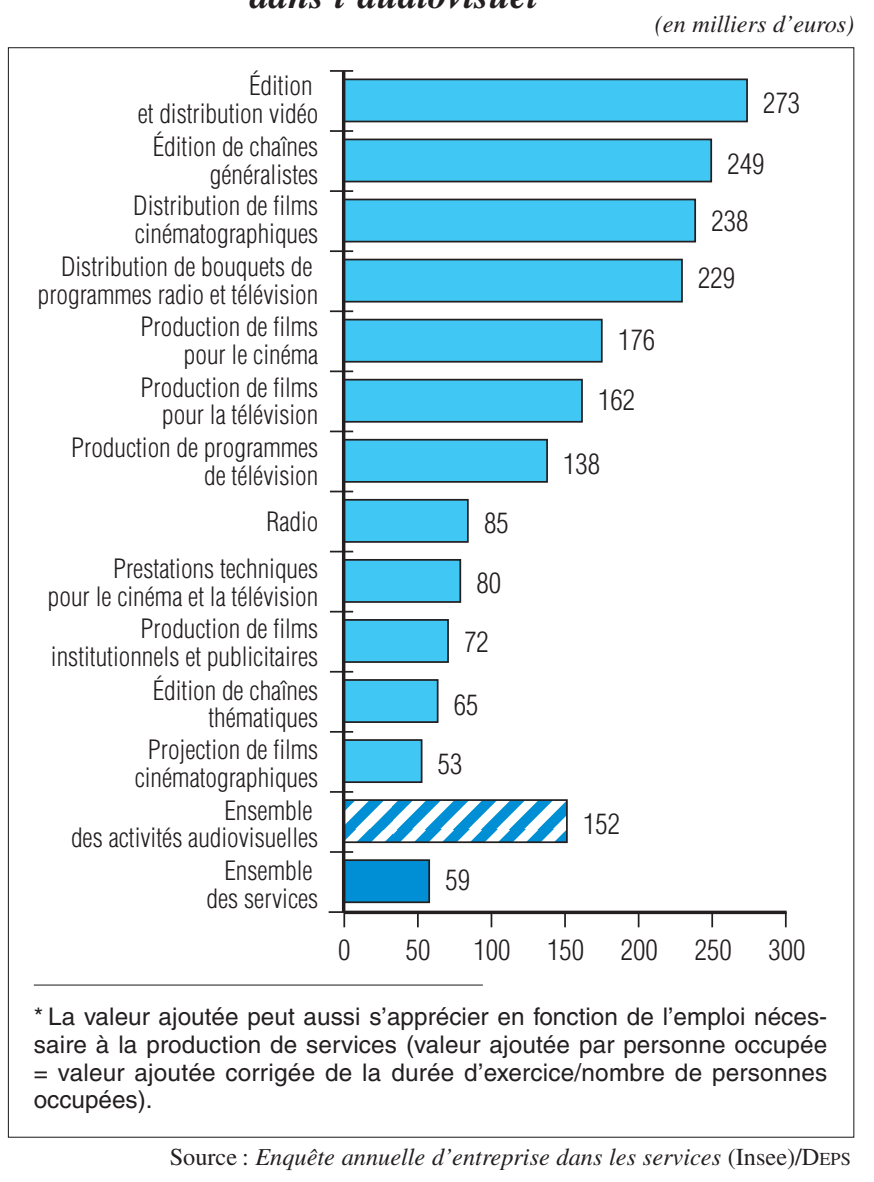

\section{Part de la valeur ajoutée et de l'emploi des industries culturelles par rapport à l'ensemble des services}

La valeur ajoutée des activités audiovisuelles a représenté, en 2006, 3,6\% de la valeur ajoutée totale de l'ensemble des services. Celle de l'édition (des entreprises de 20 salariés ou plus) a été d'environ $2 \%$ (graphique 9).

\section{Graphique 9 - Contributions en termes de valeur ajoutée et d'emploi en 2006*}

\section{Exemples de contributions à l'ensemble des services}

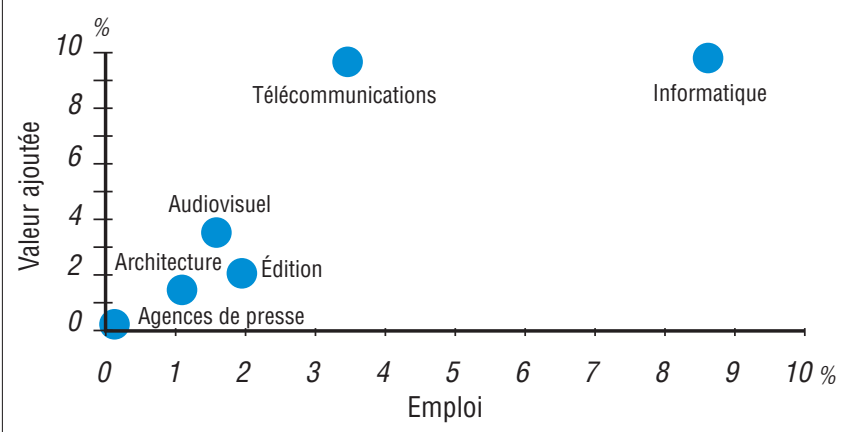

* Pour les activités de l'édition, l'emploi est estimé sur l'ensemble des entreprises; la valeur ajoutée porte sur le champ des entreprises de 20 salariés ou plus.

Sources : Enquête annuelle d'entreprise dans les services (Insee), Enquête annuelle d'entreprise dans l'industrie (Sessi)/DEPS 
Comparées à d'autres secteurs, les industries culturelles ont moins contribué à la valeur ajoutée de l'ensemble des services en 2006 que, par exemple, l'informatique ou les télécommunications (non compris La Poste). Cependant, l'écart est moins important en termes d'emploi qu'en termes de valeur ajoutée : ainsi la différence entre la part sur l'ensemble des services de l'édition et celle des télécommunications (non compris La Poste) n'est que de 1,5 point (avec respectivement $2 \%$ pour l'édition et $3,5 \%$ pour les télécommunications).

\section{Mesure}

\section{DE LA PERFORMANCE POSITIVE}

Le rapport de l'excédent brut d'exploitation $\left(\mathrm{EBE}^{6}\right)$ sur la valeur ajoutée, appelé taux de marge, mesure la rentabilité en précisant la part de la valeur créée par l'entreprise qui sert à rémunérer les apporteurs de capitaux et à renouveler le capital investi (tableau 4).

Le taux de marge des entreprises de l'édition de 20 salariés ou plus s'est fixé à $30 \%$ en 2006 ; celui de l'audiovisuel, sur un champ plus large correspondant à l'ensemble des entreprises, à $45 \%$. Comparé à l'ensemble des services dont le taux de marge a été de $19 \%$, les industries culturelles ont un taux de marge significatif.

Les écarts sont toutefois importants entre activités. Ainsi, le taux de marge des entreprises de 20 salariés ou plus du disque $(56 \%)$ est très nettement supérieur à celui des journaux (7\%) (graphique 10). Dans le sous-secteur du cinéma et de la vidéo, l'édition et la distribution vidéo ( $83 \%$ ) se démarquent des activités de projection de films cinématographiques $(18 \%)$. Les activités télévisuelles ont toutes un taux de marge supérieur à celui des services (de $23 \%$ pour l'édition de chaînes thématiques jusqu'à $72 \%$ pour la distribution de bouquets de programmes radio et télévision) (graphique 11).
Graphique 10 - Taux de marge des activités de l'édition en 2006 dans le champ des entreprises de 20 salariés ou plus

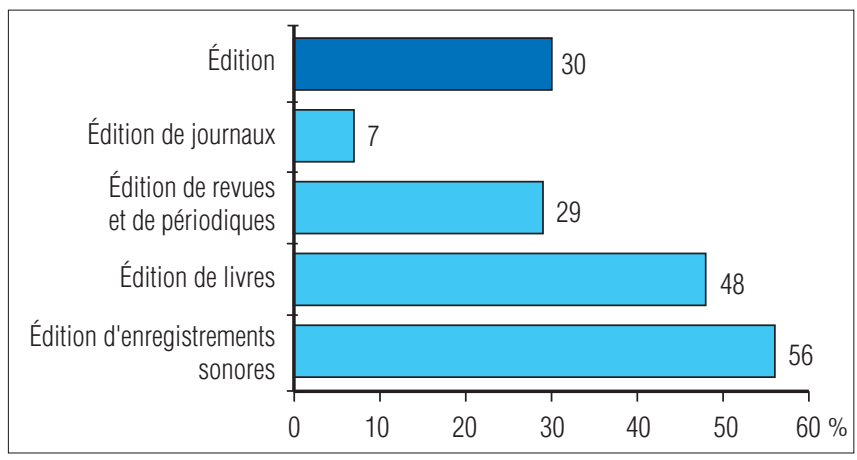

Source : Enquête annuelle d'entreprise dans l'industrie (Sessi)/DEPS

\section{Graphique 11 - Taux de marge des activités de l'audiovisuel et des agences de presse en 2006 pour l'ensemble des entreprises}

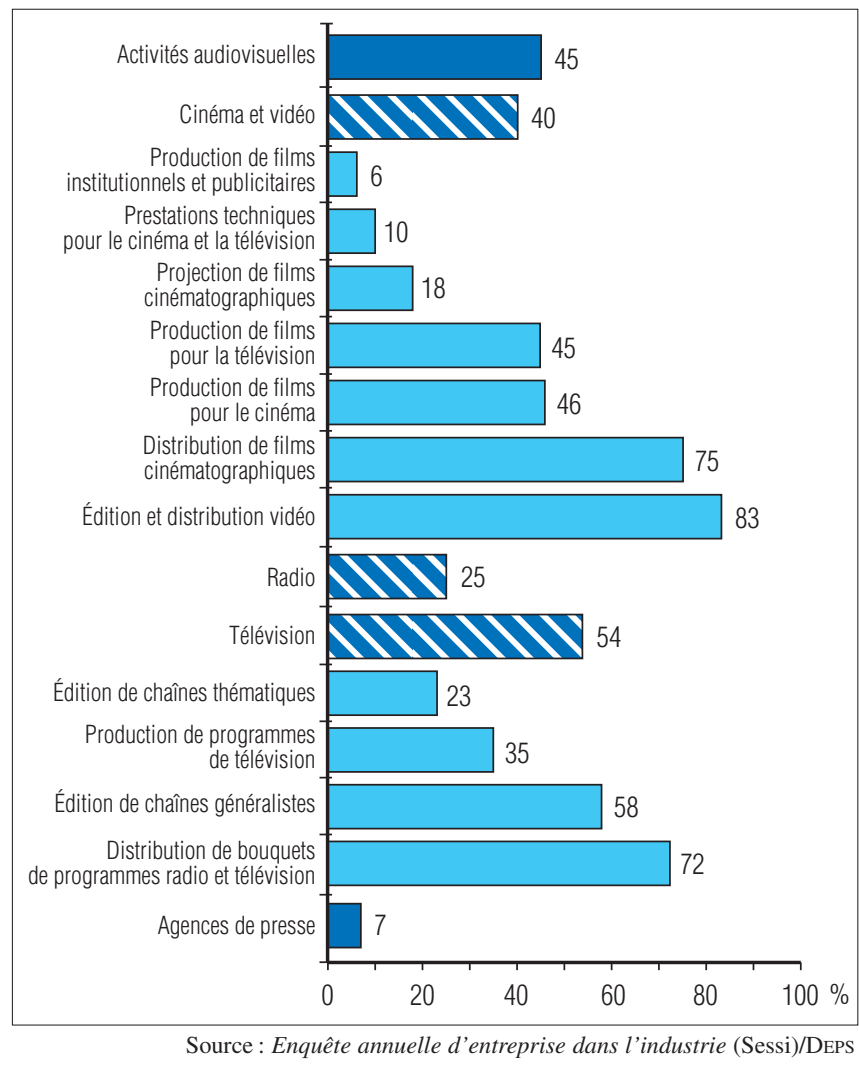

Tableau 4 - Indicateurs économiques des industries culturelles en 2006

\begin{tabular}{|c|c|c|c|c|c|}
\hline & Nombre d'entreprises & $\begin{array}{c}\text { Nombre de salariés } \\
(E T P)\end{array}$ & $\begin{array}{c}\text { CA } \\
\text { (millions d'euros) }\end{array}$ & $\begin{array}{c}\text { VA } \\
\text { (millions d'euros) }\end{array}$ & $\begin{array}{c}\mathrm{EBE} \\
\text { (millions d'euros) }\end{array}$ \\
\hline Édition & $(12107)$ & (82 929) & $(20178)$ & $6058^{*}$ & $1812^{*}$ \\
\hline $\begin{array}{l}\text { Activités audiovisuelles } \\
\text { Agences de presse }\end{array}$ & $\begin{array}{l}9970 \\
1373\end{array}$ & $\begin{array}{r}67775 \\
6241\end{array}$ & $\begin{array}{r}21999 \\
811\end{array}$ & $\begin{array}{r}10346 \\
497\end{array}$ & $\begin{array}{r}4662 \\
34\end{array}$ \\
\hline Ensemble des services & 838427 & 4208039 & 584058 & 290694 & 53839 \\
\hline
\end{tabular}

Sources : Enquête annuelle d'entreprise dans les services (Insee), Enquête annuelle d'entreprise dans l'industrie (Sessi)/DEPS

6. L'excédent brut d'exploitation (EBE) égale la valeur ajoutée diminuée des frais de personnel et des impôts liés à la production et augmentée des subventions sur les produits. Cet excédent vient notamment rémunérer les actionnaires (dividendes) et l'épargne brute de l'entreprise dont l'importance influe sur sa capacité d'investissement productif. 


\section{Méthodologie, sources et définitions}

1. Sources: les résultats sont essentiellement issus de deux enquêtes annuelles d'entreprises (EAE) dont l'Insee assure la réalisation et la coordination nationale lorsqu'elles sont effectuées par différents ministères:

- l'enquête menée auprès des entreprises de services (EAE Services) par l'Institut national de la statistique et des études économiques (Insee);

- l'enquête menée auprès des entreprises de l'industrie (EAE Industrie) par le Service des études et des statistiques industrielles (Sessi).

Toutes les entreprises du champ des industries culturelles de plus de 20 salariés sont interrogées par les EAE. Le seuil de 20 salariés, qui sépare les micro et petites entreprises des plus grandes, est le plus pertinent pour l'analyse.

Des estimations ont été réalisées par le DEPS pour appréhender l'activité des entreprises de moins de 20 salariés. Ces estimations figurent entre parenthèses. Elles utilisent des sources complémentaires de l'Insee comme le Système unifié des statistiques d'entreprises (Suse - base de données entreprises Alisse) ainsi que la démographie des entreprises du répertoire Sirene (Système informatique pour le répertoire des entreprises et des établissements).
2. Objectifs des EAE : établir des statistiques sur la structure des entreprises, la diversité et l'évolution des activités, les facteurs de production mis en œuvre, leurs performances économiques et leur compétitivité au niveau de l'investissement et de l'emploi, en satisfaisant au règlement communautaire européen sur les statistiques structurelles, et pour répondre aux besoins d'analyse macro-économique (comptes nationaux notamment) et aux analyses sectorielles.

3. Champs des EAE: l'EAE Industrie s'adresse à toutes les entreprises françaises manufacturières de 20 salariés ou plus, hors industries agricoles et alimentaires : les secteurs couverts sont ceux de l'énergie, des biens de consommation, des biens intermédiaires, de l'automobile et des biens d'équipement. L'édition imprimée et l'édition phonographique relèvent de ces EAE. Les autres industries culturelles relèvent des EAE Services qui concernent les entreprises dont l'activité principale exercée (APE) relève, dans la nomenclature d'activités française (NAF), des activités immobilières, des services aux entreprises et des services aux particuliers. Celles-ci sont annuelles et exhaustives pour les entreprises de plus de 30 salariés et d'au moins 5 millions d'euros de chiffre d'affaires annuel ; elles sont réalisées par alternance une année sur deux pour les entreprises en deçà de ces seuils (2005 : services aux entreprises et 2006 : services aux particuliers).

NB: en 2009, l'Insee abandonnera les enquêtes annuelles d'entreprises sous leur forme actuelle, au profit d'une exploitation systématique des sources fiscales, sociales et douanières; pour les variables qui ne peuvent être couvertes ainsi (variables sectorielles, ventilation du chiffre d'affaires par branche, etc.), de nouvelles enquêtes sectorielles annuelles (ESA) seront menées. 